\title{
Infección por Hysterothylacium geschei (Nematoda: Anisakidae) en peces del lago Panguipulli, Chile ${ }^{\#}$
}

\author{
Infection by Hysterothylacium geschei (Nematoda: Anisakidae) in fish \\ of Panguipulli Lake, Chile

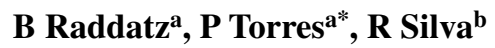 \\ anstituto de Parasitología, Facultad de Medicina, Edificio de Ciencias Biomédicas, Campus Isla Teja, \\ Universidad Austral de Chile, Valdivia, Chile. \\ bInstituto de Anatomía, Histología y Patología, Edificio de Ciencias Biomédicas, Campus Isla Teja, \\ Universidad Austral de Chile, Valdivia, Chile.
}

\begin{abstract}
SUMMARY
The objectives of the present study were: 1) to identify host fish of Hysterothylacium geschei in Lake Panguipulli, (39 $\left.43^{\circ} \mathrm{S}, 72^{\circ} 13^{\prime} \mathrm{W}\right)$, Chile, 2) to determine prevalence, mean intensity, and mean abundance of infection distributed by length and sex in each host, and 3) to determine the proportion of different developmental stages in each host. During September 2006 and January, May, and July 2007 the following fish were examined: 44 Odontesthes mauleanum (Atherinopsidae), 48 Basilichthys australis (Atherinopsidae), 25 Percichthys trucha (Percichthyidae), all natives, and 77 rainbow trout, Oncorhynchus mykiss (Salmonidae), the latter being an introduced species. All examined fish species presented infection by H. geschei and B. australis with $O$. mykiss being new hosts to $H$. geschei. The infection was present in 4, 3, and 1 of 4 samplings of $O$. mauleanum, O. mykiss and B. australis, respectively, and in 2 of 3 samplings of $P$. trucha. Third and fourth stage-larvae were present in the 4 hosts; males were identified in 3 hosts, except in $P$. trucha. The occurrence of gravid females was evident only in $O$. mauleanum. The prevalence $(63.6 \%)$, and mean abundance (9.8) were significantly greater in $O$. mauleanum compared to the other hosts (prevalence: $8.3 \%-16 \%$, mean abundance: $0.2-0.7)$. The mean intensity (15.3) was significantly higher in $O$. mauleanum compared to P. trucha (1.3) and O. mykiss (1.9). Mean intensity was similar among O. mauleanum and B. australis (8.8). The prevalence, mean intensity, and mean abundance of infection were similar between sexes in all hosts. The abundance of infection was positively correlated with length for $O$. mauleanum and $O$. mykiss, which was not evident in P. trucha and B. australis. The L3 recruitment in $O$. mauleanum occurred in all the months of the study.
\end{abstract}

Key words: Hysterothylacium geschei, native fish, introduced trout, Panguipulli Lake.

\section{RESUMEN}

Los objetivos del presente estudio fueron: 1) identificar peces hospederos de Hysterothylacium geschei en el lago Panguipulli ( $\left.39^{\circ} 43^{\prime} \mathrm{S}, 72^{\circ} 13^{\prime} \mathrm{W}\right)$, Chile, 2) determinar la prevalencia, intensidad media y abundancia media de infección en cada hospedero, distribuidos por longitud estándar y sexo y 3) determinar la proporción de los distintos estadios de desarrollo en cada hospedero. Durante septiembre de 2006 y enero, mayo y julio de 2007 los siguientes peces fueron examinados: 44 Odontesthes mauleanum (Atherinopsidae), 48 Basilichthys australis (Atherinopsidae), 25 Percichthys trucha (Percichthyidae), todas especies nativas, y 77 truchas arco iris introducidas, Oncorhynchus mykiss (Salmonidae). Todas las especies de peces examinadas presentaron infección por $H$. geschei. Basilichthys australis y $O$. mykiss son nuevos hospederos para $H$. geschei. La infección estuvo presente en 4,3 y 1 de los 4 muestreos de $O$. mauleanum, $O$. mykiss y $B$. australis, respectivamente, y en 2 de los 3 muestreos en que se capturó $P$. trucha. Larvas del tercero y cuarto estadio estuvieron presentes en los 4 hospederos, los machos fueron observados en 3 hospederos, excepto en $P$. trucha. La presencia de hembras grávidas solo fue evidente en $O$. mauleanum. La prevalencia $(63,6 \%)$ y abundancia media $(9,8)$ fueron significativamente mayores en $O$. mauleanum respecto de $P$. trucha y $O$. mykiss (prevalencia: $8,3 \%-16 \%$, abundancia media: $0,2-0,7)$. La intensidad media $(15,3)$ fue significativamente mayor en $O$. mauleanum respecto de $P$. trucha $(1,3)$ y $O$, mykiss $(1,9)$, pero resultó similar a la intensidad media en $B$. australis $(8,8)$. La prevalencia, intensidad media y abundancia media de infección fueron similares entre sexos de cada hospedero. La abundancia de infección fue positivamente correlacionada con la longitud de $O$. mauleanum y O. mykiss. El reclutamiento de las larvas del tercer estadio en $O$. mauleanum fue observado en todos los meses del estudio.

Palabras clave: Hysterothylacium geschei, peces nativos, truchas introducidas, lago Panguipulli.

\section{INTRODUCCIÓN}

El género Hysterothylacium incluye alrededor de 70 especies de nematodos de ciclo vital indirecto cuyo estadio

Aceptado: 15.05.2014.

\# Financiado parcialmente por el programa de diversidad de parásitos y zoonosis transmitidas por organismos acuáticos (DID I-201002).

* Casilla 567, Valdivia, Chile; ptorres@uach.cl adulto desarrolla en el tracto digestivo de peces marinos, de agua dulce o de estuarios (Li y col 2013). El efecto de Hysterothylacium spp. en peces ha sido escasamente investigado, sin embargo existen registros que indican que Hysterothylacium aduncum causa mortalidad en peces juveniles (Balbuena y col 2000) y que las infecciones intensas por Hysterothylacium bidentatum inducen obstrucción del tracto digestivo (Molnar y col 2006). Hysterothylacium spp. han sido asociadas a cuadros alérgicos (Valero y col 
2003) e Hysterothylacium aduncum a un caso de infección humana en Japón (Yagi y col 1996).

En América del sur han sido descritas 9 especies de Hysterothylacium (Knoff y col 2012), de ellas tres se desarrollan en peces nativos de agua dulce: Hysterothylacium rhamdiae en Rhamdiae sapo en ríos del distrito de Buenos Aires, Argentina (Brizzola y Tanzola, 1995), Hysterothylacium patagonense en Percichthys trucha en el lago Aluminé, Patagonia argentina (Moravec y col 1997) e Hysterothylacium geschei en Odontesthes mauleanum en el lago Panguipulli, en el sur de Chile (Torres y col 1998). En dichas especies no se han realizado estudios suficientes acerca del espectro de hospederos y su prevalencia, intensidad y abundancia de infección. Lo anterior, debido a la complejidad de sus ciclos de vida que incluyen el desarrollo de distintos estadios, en ambientes acuáticos y en diferentes hospederos, como adulto y larvas del primer (L1), segundo (L2), tercer (L3) y cuarto (L4) estadio (Moravec 1994, Anderson 2000). En el ciclo de vida de Hysterothylacium spp., basado esencialmente en Hysterothylacium aduncum (Koie 1993, Klimpel y Ruckert 2005), los huevos eliminados con las heces del hospedero definitivo desarrollan L1, L2 y L3 en el agua. La L3 al ser ingerida por un crustáceo hospedero intermediario (copépodos, anfípodos, isópodos, misidáceos) eclosiona en su tracto digestivo, localizándose en el hemocele. Distintos macroinvertebrados (por ejemplo, quetognatos, poliquetos, ctenóforos y ofiuroideos) y pequeños peces se pueden infectar al consumir crustáceos, actuando como hospedero intermediario o paraténico. Las L3 ingeridas con invertebrados o peces, con más de $3 \mathrm{~mm}$ de longitud, desarrollan a L4 y adultos en el pez hospedero definitivo, sin embargo, las L3 de 2-3 mm no se desarrollan, ingresando a la pared intestinal o cavidad corporal donde encapsulan. Las L3 con menos de $2 \mathrm{~mm}$ no sobreviven en el hospedero definitivo.

En $H$. rhamdiae solo se ha descrito su estado adulto (Brizzola y Tanzola 1995), en H. patagonense se han identificado adultos, L3 y L4 en $P$. trucha y solo L3 en truchas introducidas, Oncorhynchus mykiss, Salvelinus fontinalis y Salmo trutta (Moravec y col 1997). En H. geschei se han descrito L3, L4, y adultos en $O$. mauleanum y solamente L3 en $P$. trucha (Torres y col 1998).

Entre las especies de Hysterothylacium de peces de agua dulce del hemisferio norte, $H$. analarum se trasmite a Lepomis gibbosus al consumir copépodos, Acanthocyclops vernalis, infectados con L3, alcanzando su mayor intensidad de infección en el verano, coincidiendo con el período de reclutamiento de las L3, que se extiende hasta otoño en Ontario, Canadá (Rye y Baker 1992). Hysterothylacium bidentatum infecta a su hospedero (Acipenser sp.) por consumo de crustáceos anfípodos infectados con L4, en el que mudan y dan origen al adulto en el estómago, observándose las mayores prevalencias en verano y otoño en el río Volga (Moravec 1994). En Hysterothylacium haze el hospedero definitivo (Acanthogobius flavimanus) se encuentra en bahías y estuarios alcanzando ocasionalmente los ríos; adquiere las larvas infectantes existentes en el agua o en hospederos intermediarios/paraténicos, como poliquetos (Nereis sp.), anfípodos (Ampithoe valida, Corophium uenoi, Grandidierella japonica) o misidáceos (Neomysis japonica) (Yoshinaga y col 1989). Su prevalencia alcanza el $70 \%$ en A. flavimanus en otoño en la bahía de Tokio (Yoshinaga y col 1987, 1989).

Hysterothylacium aduncum es cosmopolita y su ciclo vital es el más estudiado entre la especies congenéricas; sin embargo, infecta a peces marinos y peces migratorios, presentándose en estos últimos solo durante su retorno al agua dulce (Moravec 1994). No hay evidencias que el ciclo de $H$. aduncum pueda suceder en ecosistemas dulceacuícolas, en ausencia de contacto con el mar (Moravec 1994). Presenta un amplio espectro de peces hospederos en el mundo y en Chile se le ha registrado en cultivos marinos de O. mykiss, Oncorhynchus kisutch y Salmo salar y en peces silvestres marinos y de estuarios, como Merluccius australis, Hypsoblennius sordidus, Normanichthys crokeri, Strangomera bentincki, Odontesthes regia, Eleginops maclovinus, Trachurus murphy, Prolatilus jugularis y Nezumia pulchella (González y Carvajal 1995, Sepúlveda y col 2004, Salinas y col 2008, Torres y col 2010). En Chile, de 13 especies de peces marinos y de estuarios comercializados frescos para su consumo y de 3 especies de salmónidos cultivados (O. mykiss, S. salar y $O$. kisutch) solo en un ejemplar de merluza de cola, Macrouronus magellanicus, se encontró una larva de Hysterothylacium sp. en los músculos, a diferencia de otros anisákidos más frecuentes en peces marinos y causantes de zoonosis como Pseudoterranova spp. y Anisakis spp. (Torres y col 2000, 2010, 2014).

La introducción exitosa de trucha arco iris en Chile se logró a partir de la importación de ovas desde Hamburgo, Alemania, en 1905 (Golusda 1927). Las poblaciones de trucha arco iris y otros peces introducidos en distintos países han podido adquirir infecciones por parásitos, comúnmente generalistas a partir de la fauna local, pudiendo tener un serio impacto ecológico (Kennedy y Bush 1994, Poulin y Mouillot 2003, Kelly y col 2009). En Chile se han identificado tres especies de nematodos en $O$. mykiss: H. aduncum, Camallanus corderoi y Contracaecum rudolphii (sensu lato), todas con amplio espectro de hospederos nativos (Torres 2012).

Los objetivos del presente estudio fueron: 1) identificar peces hospederos de $H$. geschei en el lago Panguipulli (39 $\left.43^{\prime} \mathrm{S}, 72^{\circ} 13^{\prime} \mathrm{W}\right)$, Chile, 2) determinar la prevalencia e intensidad y abundancia media de infección en cada hospedero distribuidos por su longitud estándar y sexo, y 3) determinar la proporción de los distintos estadios de desarrollo en cada hospedero.

\section{MATERIAL Y MÉTODOS}

Los peces fueron capturados durante los meses de septiembre de 2006 y enero, mayo y julio de 2007 en el 
sector este del lago Panguipulli, utilizando redes de 25, 32 y $45 \mathrm{~mm}$ de abertura, colocadas por dos noches consecutivas. Los peces fueron trasladados en hielo al laboratorio para realizar su disección, dentro de las seis horas de su captura. Los peces identificados, su longitud estándar y sexo se indican en el cuadro 1.

Durante la disección de los peces se separó estómago e intestino, estos fueron abiertos longitudinalmente en placas de Petri con solución salina $(\mathrm{NaCl} 0,15 \mathrm{M})$, sus superficies externa e interna fueron observadas en un microscopio estereoscópico raspando la mucosa y colectando los nematodos encontrados. El hígado, bazo, páncreas, vejiga natatoria, gónadas, mesenterios y corazón fueron examinados y desmenuzados bajo microscopio estereoscópico en placas de Petri con solución salina. Los músculos fueron examinados mediante las técnicas de candling y compresión en placas siguiendo a Torres y Puga (2011). Los nematodos aislados fueron fijados en formol-salino y diafanizados en lactofenol para su estudio morfológico y métrico en microscopio de luz. La identificación de los estadios de desarrollo de $H$. geschei se basan, en general, en las características descritas por Torres y col (1998). Adicionalmente, algunos ejemplares fueron fijados en glutaraldehído, procesándolos de acuerdo con Torres y Soto (2004), para observar en un microscopio LEO 420 (Leo Electron Microscopy, Cambridge, UK).

Los términos de prevalencia, intensidad media y abundancia media se aplicaron de acuerdo con Margolis y col (1982). Los análisis de prevalencia incluyeron las pruebas de $\chi^{2}$ con corrección de Pearson para tres o más muestras, para pares de muestras se aplicó la misma prueba con corrección de Yates o la prueba de Fisher, esta última cuando alguna frecuencia esperada fue < de 5 (Zar 1999). Para comparar la abundancia media de infección se aplicó la prueba de varianza de Kruskall-Wallis para más de dos muestras cuyo tamaño fue mayor de cinco. La prueba de U de Mann-Whitney fue aplicada para la comparación de intensidad y abundancia media en pares de muestras (Siegel 1972). Los análisis de correlación se realizaron mediante la prueba de rangos de Spearman $\left(r_{s}\right)$ (Zar 1999). Para la mayoría de las pruebas $\mathrm{P}<0,05$ fue considerada estadísticamente significativa. Para la prueba de U de Mann-Whitney el valor de $\mathrm{P}$ fue ajustado, según Bonferroni (Sachs 1968), en las comparaciones de tres o más pares de muestras.

\section{RESULTADOS}

Hysterothylacium geschei estuvo presente en el intestino de las cuatro especies de peces examinadas. Las características morfológicas e intervalos de las medidas utilizadas para la diferenciación de los distintos estadios de desarrollo (adultos, L3, y L4) se reseñan en el cuadro 2 y en la figura 1. La infección fue determinada en 4,3 y 1 de los cuatro muestreos de O. mauleanum, O. mykiss y $B$. australis, respectivamente, y en dos de los tres muestreos en que se capturó ejemplares de $P$. trucha. La prevalencia y abundancia media de infección fueron significativamente mayores en $O$. mauleanum, respecto de $P$. trucha y $O$. mykiss; en estas dos últimas especies ambos descriptores resultaron similares (cuadro 3). La intensidad media de infección resultó significativamente mayor en $O$. mauleanum respecto de $P$. trucha y $O$. mykiss, pero fue similar con la intensidad media en $B$. australis. La prevalencia, intensidad media y abundancia media fueron similares al comparar la infección en peces de distinto sexo en $O$. mauleanum, B. australis, $P$. trucha y O. mykiss (cuadro 4). La abundancia de infección estuvo significativamente correlacionada con la longitud estándar de O. mauleanum $\left(\mathrm{r}_{\mathrm{s}}=0,6 ; \mathrm{P}<0,05 ; \mathrm{n}=44\right)$ y $O$. mykiss $\left(\mathrm{r}_{\mathrm{s}}=0,3 ; \mathrm{P}<0,05 ; \mathrm{n}=77\right)$. En P. trucha $\left(\mathrm{r}_{\mathrm{s}}=0,2 ; \mathrm{P}>\right.$ $0,05 ; \mathrm{n}=25)$ y $B$. australis $\left(\mathrm{r}_{\mathrm{s}}=0,3 ; \mathrm{P}>0,05 ; \mathrm{n}=48\right)$ no se observó correlación significativa entre la abundancia de infección y la longitud del hospedero.

El reclutamiento de L3 así como la presencia de L4 fueron evidentes en los cuatro hospederos; las L3 mudando a L4 y los ejemplares machos fueron observados en la

Cuadro 1. Especies, sexo y longitud estándar de los peces examinados del lago Panguipulli, Chile. Species, sex, and standard length of the examined fish from Panguipulli Lake, Chile.

\begin{tabular}{|c|c|c|c|c|}
\hline \multirow{2}{*}{ Peces (número) } & \multicolumn{3}{|c|}{ Sexo } & \multirow{2}{*}{ Longitud estándar $(\mathrm{cm})$} \\
\hline & Machos & Hembras & $\mathrm{ND}^{\mathrm{a}}$ & \\
\hline \multicolumn{5}{|l|}{$\begin{array}{l}\text { Nativos } \\
\text { Atherinopsidae }\end{array}$} \\
\hline Odontesthes mauleanum (44) & 8 & 33 & 3 & $27 \pm 5(18-36)$ \\
\hline Basilichthys australis (25) & 9 & 38 & 1 & $27 \pm 5(22-37)$ \\
\hline \multicolumn{5}{|l|}{ Percichthyidae } \\
\hline Percichthys trucha (25) & 9 & 12 & 4 & $36 \pm 7(16-43)$ \\
\hline \multicolumn{5}{|l|}{$\begin{array}{l}\text { Introducido } \\
\text { Salmonidae }\end{array}$} \\
\hline Oncorhynchus mykiss (77) & 21 & 40 & 16 & $37 \pm 9(19-70)$ \\
\hline
\end{tabular}

${ }^{\mathrm{a}} \mathrm{ND}=$ no determinado. 
mayoría de los hospederos, excepto en P. trucha (cuadro 5). Las hembras fueron identificadas en todos los hospederos, pero las hembras grávidas con huevos solo se encontraron en $O$. mauleanum (cuadro 5).

En O. mauleanum, las L3 fueron observadas en todo los muestreos, con mayor proporción en marzo y junio, las L4 y las hembras inmaduras predominaron en septiembre, mientras que las hembras grávidas y machos predominaron en enero (cuadro 6). La prevalencia, intensidad media y abundancia media de infección fueron similares en los 4 muestreos (cuadro 7).

\section{DISCUSIÓN}

Hysterothylacium geschei se desarrolla en las tres especies de peces nativos examinadas en el lago Panguipulli, incluidas en las familias Atherinopsinae (O. mauleanum y B. australis) y Percichthyidae (P. trucha), así como en la trucha arco iris introducida. Basilichthys australis y $O$. mykiss se detectan por primera vez como hospederos de $H$. geschei y en $P$. trucha se identifican por primera vez L4 y hembras inmaduras. Anteriormente se habían registrado L3 de Hysterothylacium de una especie no identificada en $O$. mauleanum con prevalencias de $28,6 \%$ y $3,6 \%$ e intensidades medias de uno en el lago Calafquén y río Calle Calle, respectivamente, así como en $B$. australis en el lago Riñihue con prevalencia de $1,7 \%$ e intensidad media de uno (Torres y col 1988). También en 211 adultos de $O$ mykiss capturados en 8 lagos situados entre $39^{\circ} 03^{\prime}$ y $41^{\circ} 05^{\prime}$ de latitud sur solo se identificó L3 de Hysterothylacium sp. en el lago Ranco, con prevalencia de $3 \%$ (Torres 1995). Probablemente dichos hallazgos pudieron corresponder a $H$. geschei, descrita posteriormente (Torres y col 1998).

En el lago Panguipulli, O. mauleanum sería el hospedero principal de $H$. geschei, ya que su prevalencia, intensidad media y abundancia media fueron de 4 a 8,2 a 11 , y de 14 a 49 veces mayor que en los demás hospederos identificados, respectivamente, aunque la intensidad media no mostró diferencias significativas entre O. mauleanum y $B$. australis. Además, O. mauleanum fue el único hospedero que presentó hembras grávidas en los cuatro muestreos, con mayor proporción en enero, correspondiente a la estación de verano en que las aguas superficiales del lago alcanzan hasta $21^{\circ} \mathrm{C}$ (Soto y Campos 1996). Lo anterior podría favorecer el desarrollo de las L3 en menor tiempo en los huevos del parásito respecto de los meses en que declina la temperatura superficial del agua en el lago. En $H$. analarum la larva infectante se desarrolla en 48 h a $20-23{ }^{\circ} \mathrm{C}$ (Rye y Baker 1992), en H. haze en 7 días a $20^{\circ} \mathrm{C}$ (Yoshinaga y col 1989) y en $H$. aduncum en 5 y 14 días a $20{ }^{\circ} \mathrm{C}$ y $5{ }^{\circ} \mathrm{C}$, respectivamente (Koie 1993). El desarrollo más acelerado de los huevos y formación de las L3 en verano coincide además con el incremento de las poblaciones de zooplancton y algunas especies de copépodos en lagos del sur de Chile (Campos y col 1992, Campos 2000), potenciales hospederos de L3 de Hysterothylacium spp. La mayor proporción de hembras grávidas en meses de verano también ha sido observada en distintos hospederos de H. aduncum (Fagerholm 1982, Andersen 1993), H. analarum (Rye y Baker 1992) e $H$. bidentatum (Moravec 1994) en el hemisferio norte.

Cuadro 2. Intervalos de características métricas ( $\mathrm{mm}$ ) de Hysterothylacium geschei en peces del lago Panguipulli, Chile. Intervals of metric characteristics (mm) of Hysterothylacium geschei in fish from Panguipulli Lake, Chile.

\begin{tabular}{|c|c|c|c|c|c|}
\hline \multirow[b]{2}{*}{ Características } & \multirow{2}{*}{$\frac{\text { L3 }}{(n=9)^{\mathrm{a}}}$} & \multirow{2}{*}{$\frac{\mathrm{L} 4}{(\mathrm{n}=28)^{\mathrm{b}}}$} & \multicolumn{2}{|c|}{ Hembras } & \multirow{2}{*}{$\frac{\text { Machos }}{(\mathrm{n}=20)^{\mathrm{b}}}$} \\
\hline & & & $\begin{array}{c}\text { inmaduras } \\
(\mathrm{n}=18)^{\mathrm{b}}\end{array}$ & $\begin{array}{l}\text { grávidas } \\
(\mathrm{n}=12)^{\mathrm{c}}\end{array}$ & \\
\hline Largo corporal & $15,5-26,3$ & $17-27$ & $21,5-42,5$ & $35,2-73,5$ & $28,6-49,3$ \\
\hline Ancho & $0,4-0,5$ & $0,4-0,6$ & $0,5-0,9$ & $0,7-1,5$ & $0,6-1,4$ \\
\hline Interlabios & - & $0,04-0,1$ & $0,04-0,12$ & $0,07-0,2$ & $0,06-0,1$ \\
\hline \multicolumn{6}{|l|}{ Distancia boca } \\
\hline -anillo nervioso & $0,1-0,5$ & $0,3-0,6$ & $0,4-0,6$ & $0,6-1,4$ & $0,5-0,9$ \\
\hline -poro excretor & $0,2-0,7$ & $0,4-0,9$ & $0,5-0,7$ & $1-1,5$ & $0,7-1,9$ \\
\hline -vulva & - & - & $8-13,5$ & $11,3-26$ & - \\
\hline Esófago & $0,7-2,8$ & $1,9-3,1$ & $2,2-3,3$ & $3,5-8,3$ & $2,9-6,2$ \\
\hline Ventrículo (V) & $0,04-0,2$ & $0,1-0,2$ & $0,1-0,2$ & $0,3-0,5$ & $0,1-0,3$ \\
\hline Apéndice del V & $0,6-1,2$ & $0,7-1,3$ & $0,8-1,5$ & $1,2-2,6$ & $0,9-1,8$ \\
\hline Ciego intestinal & $0,4-1$ & $0,7-1,3$ & $0,9-1,5$ & $1,3-2,6$ & $0,7-1,6$ \\
\hline Cola & $0,07-0,2$ & $0,1-0,3$ & $0,1-0,3$ & $0,3-0,5$ & $0,1-0,2$ \\
\hline Espículas & - & - & - & - & $0,8-2,1$ \\
\hline Huevos & - & - & - & $0,06-0,07$ & - \\
\hline
\end{tabular}

Material aislado en: Odontesthes mauleanum (OM), Basilichthys autralis (BA), Percichthys trucha (PT) y Oncorhynchus mykiss ${ }^{\mathrm{a}}(\mathrm{OMY})$, en OM, BA y $\mathrm{OMY}^{\mathrm{b}}$, en $\mathrm{OM}^{\mathrm{c}}$. 

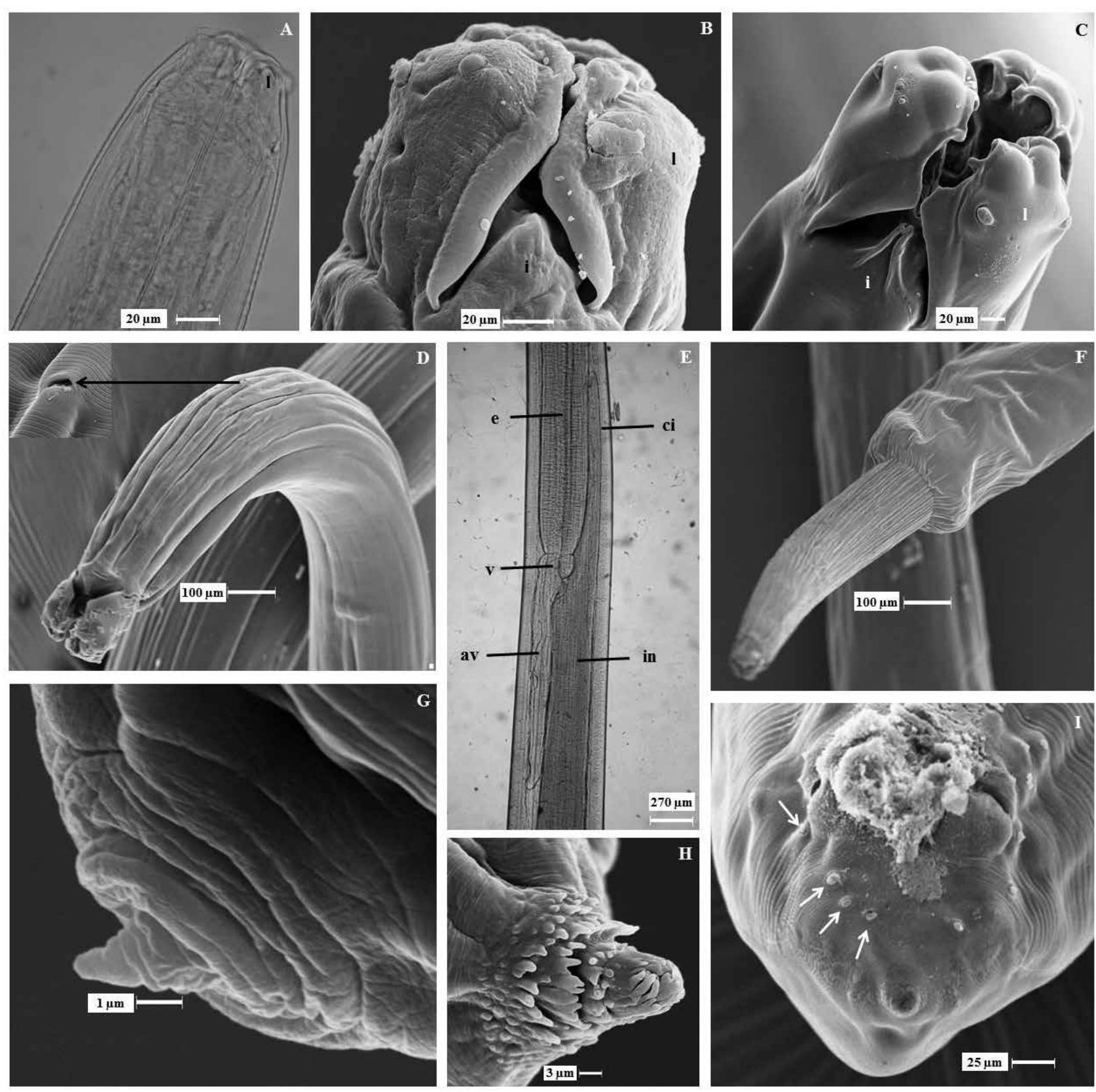

Figura 1. Hysterothylacium geschei: A) Región cefálica (RC): larva del tercer estadio (L3) con microscopio de luz (ML). B) RC: larva del cuarto estadio (L4) con microscopio electrónico de barrido (MEB). C) RC: hembra con MEB. D) Poro excretor (flecha): hembra con MEB. E) Región esófago-intestinal: hembra con ML. F) L3 mudando a L4 con MEB. G) Cola (CO): L3 con MEB. H) CO con espinas: hembra con MEB. I) CO: macho con 4 pares de papilas postanales (flechas). $1=$ labios, $\mathrm{i}=$ interlabio, in = intestino, e = esófago, $\mathrm{ci}=$ ciego intestinal, av = apéndice ventricular

Hysterothylacium geschei: A) Cephalic region (CR): third stage-larva (L3) with light microscope (LM). B) CR: fourth stage larva (L4) with scanning electron microscope (SEM). C) CR: female with SEM. D) Excretory pore (arrow): female with SEM. E) Esophagus-intestinal region: female with LM. F) L3 molting to L4 with SEM. G) Tail (TA): L3 with SEM. H) TA: with spines: female with SEM. I) TA: male with 4 pairs of post anal papillae (arrows). $1=$ lips, $\mathrm{i}=$ interlabium, in $=$ intestine, $\mathrm{e}=$ esophagus, $\mathrm{ci}=$ intestinal cecum, av $=$ ventricular appendage.

La mayor prevalencia, intensidad media y abundancia media de infección por H. geschei en $O$. mauleanum, respecto de los demás hospederos, podría estar relacionado con diferencias en su dieta, mayor frecuencia o abundancia en el consumo de presas/ hospederos intermediarios o paraténicos, tamaño de las presas o diversidad de presas consumidas.
La dieta de $O$. mauleanum está basada principalmente en crustáceos decápodos (Aegla spp.), anfípodos y misidáceos (Campos 1985, Klink y Eckmann 1985, Ruiz y Marchant 2004). Al menos Aegla denticulata ha sido registrada como hospedero de larvas de Hysterothylacium sp. en el lago Rupanco con prevalencia de 0,2\% (Torres y Jara 1986). 
Cuadro 3. Prevalencia, intensidad media y abundancia media de infección por Hysterothylacium geschei en peces del lago Panguipulli, Chile.

Prevalence, mean intensity, and mean abundance of infection by Hysterothylacium geschei in fish from Panguipulli Lake, Chile.

\begin{tabular}{lccc}
\hline Peces & Prevalencia $^{\mathrm{a}}$ & Intensidad $^{\mathrm{b}}$ & Abundancia $^{\mathrm{c}}$ \\
\hline Odontesthes mauleanum $(\mathrm{OM})$ & $28 / 44$ & $15,3 \pm 24,1$ & $9,8 \pm 20,6$ \\
& $(64 \%)$ & $(1-94)$ & $0,7 \pm 4,6$ \\
Basilichthys australis (BA) & $4 / 48$ & $(1-32)$ & $0,2 \pm 0,5$ \\
Percichthys trucha $(\mathrm{PT})$ & $(8 \%)$ & $1,3 \pm 0,5$ & $(1-2)$ \\
& $4 / 25$ & $1,9 \pm 1,2$ & $0,3 \pm 0,8$ \\
Oncorhynchus mykiss $(\mathrm{OMY})$ & $(16 \%)$ & $(1-5)$ & \\
& $(11 / 77$ & & $0,3)$ \\
\hline
\end{tabular}

a Peces infectados / examinados (\%). Comparaciones: OM/BA/PT/OMY $\left(\chi^{2}=48,9^{*}\right), \mathrm{OM} / \mathrm{BA}\left(\chi^{2}=28,6^{*}\right), \mathrm{OM} / \mathrm{PT}\left(\chi^{2}=12,7^{*}\right), \mathrm{OM} / \mathrm{OMY}$ $\left(\chi^{2}=29 *\right)$, BA/PT, PT/OMY (Prueba de Fisher NS).

b Media \pm desviación estándar (mínima-máxima). Prueba de U-Mann Whitney (UMW) corregida por Bonferroni. Comparaciones: OM/BA ( $\mathrm{Z}=2,0)$ $\mathrm{OM} / \mathrm{PT}\left(\mathrm{Z}=2,6^{*}\right)$ OM/OMY $\left(\mathrm{Z}=3^{*}\right)$, PT/BA (U = 14: NS), PT/OMY (U = 14,5: NS), BA/OMY (U = 21: NS).

c Media \pm desviación estándar. Pruebas de Kruskall-Wallis y UMW corregida por Bonferroni. Comparaciones: BM/BA/PT/OMY $(\mathrm{H}=56,7 *), \mathrm{OM} /$ BA $\left(Z=5,6^{*}\right), \mathrm{OM} / \mathrm{PT}\left(\mathrm{Z}=4,2^{*}\right), \mathrm{OM} / \mathrm{OMY}\left(\mathrm{Z}=6,1^{*}\right), \mathrm{PT} / \mathrm{BA}(\mathrm{Z}=0,9 \mathrm{NS}), \mathrm{PT} / \mathrm{OMY}(\mathrm{Z}=0,1 \mathrm{NS}) \mathrm{BA} / \mathrm{OMY}(\mathrm{Z}=1 \mathrm{NS})$.

* Diferencias significativas $(\mathrm{P}<0,05), \mathrm{NS}=$ no significativa $(\mathrm{P}>0,05)$.

Cuadro 4. Prevalencia, intensidad media y abundancia media de infección por Hysterothylacium geschei en peces del lago Panguipulli de acuerdo con el sexo del hospedero.

Prevalence, mean intensity, and mean abundance of infection by Hysterothylacium geschei in fish from Panguipulli Lake, Chile, distributed by host sex.

\begin{tabular}{|c|c|c|c|c|}
\hline \multirow[b]{2}{*}{ Descriptores de infección / sexo } & \multicolumn{4}{|c|}{ Peces } \\
\hline & $\begin{array}{l}\text { Odontesthes } \\
\text { mauleanum }\end{array}$ & $\begin{array}{l}\text { Percichthys } \\
\text { trucha }\end{array}$ & $\begin{array}{c}\text { Basilichthys } \\
\text { australis }\end{array}$ & $\begin{array}{c}\text { Oncorhynchus } \\
\text { mykiss }\end{array}$ \\
\hline \multicolumn{5}{|l|}{ Prevalencia } \\
\hline Machos & $5 / 8(62,5)^{\mathrm{a}}$ & $1 / 9(11,1)$ & $0 / 9(0)$ & $2 / 21(9,5)$ \\
\hline Hembras & $21 / 33(63,6)$ & $3 / 12(25,0)$ & $4 / 38(10,5)$ & $7 / 40(17,5)$ \\
\hline Prueba de Fisher ${ }^{\mathrm{b}}$ & $\mathrm{P}=1^{\mathrm{b}}$ & $P=0,6$ & $P=0,6$ & $\mathrm{P}=0,5$ \\
\hline \multicolumn{5}{|l|}{ Intensidad media ${ }^{c}$} \\
\hline Machos & $11,4(2-33)^{\mathrm{c}}$ & $1(1)$ & 0 & $1(1)$ \\
\hline Hembras & $17,9(1-94)$ & $1,3(1-2)$ & $8,8(1-32)$ & $1,7(1-2)$ \\
\hline Prueba de U Mann-Whitney ${ }^{b}$ & $\mathrm{Z}=0,03$ & $\mathrm{U}=3$ & $\mathrm{ND}^{\mathrm{d}}$ & $\mathrm{U}=14$ \\
\hline \multicolumn{5}{|l|}{ Abundancia media $^{\mathrm{d}}$} \\
\hline Machos & 7,1 & 0,1 & 0 & 0,1 \\
\hline Hembras & 11,4 & 0,3 & 0,9 & 0,3 \\
\hline Prueba de U Mann-Whitney ${ }^{b}$ & $\mathrm{Z}=0,1$ & $\mathrm{U}=3$ & $Z=1$ & $Z=0,9$ \\
\hline
\end{tabular}

\footnotetext{
Peces infectados / examinados $(\%)$.

Valores no significativos $(\mathrm{P}>0,05)$.

Media (mínima - máxima).

No determinado.
}

Especies de anfípodos han sido identificadas como hospedero intermediario/paraténico en H.bidentatum, $H$. haze e H. aduncum y de misidáceos en H. haze e H. aduncum (Yoshinaga y col 1987,1989, Moravec 1994), además de decápodos (Norris y Overstreet 1976, Hurst 1984, Marcogliese 1996). Las prevalencias de infección por $H$. aduncum en crustáceos varían ampliamente, dependiendo de la especie, edad y distribución del hospedero y estación del año (Norris y Overstreet 1976). Por ejemplo, la prevalencia puede variar entre 0,3 y $100 \%$ para $H$. aduncum en el decápodo Pagurus acadianus en el este de Canadá con intensidades que suelen ser muy bajas (1-1,7 larvas por hospedero) (Marcogliese 1996). La dieta de Basilichthys australis sugiere que no consume Aegla spp., anfípodos y misidáceos, sin embargo consume copépodos (Campos 1985, Ruiz y Marchant 2004), que podrían constituir su única fuente de infección; en $H$. aduncum e $H$. analarum algunas especies de copépodos favorecen su transmisión a los peces (Norris y Overstreet 1976, Rye y Baker 1992, Marcogliese 1996). Percichthys trucha en las tallas de los peces examinados tienen como presas predominantes a Aegla spp. (72\%) y peces (22\%) (Arenas 1980), mientras 
Cuadro 5. Estadios del desarrollo de Hysterothylacium geschei identificados en peces hospederos del lago Panguipulli, Chile. Hysterothylacium geschei stages development identified in fish hosts from Panguipulli Lake, Chile.

\begin{tabular}{|c|c|c|c|c|c|c|c|c|}
\hline \multirow{3}{*}{$\begin{array}{l}\text { Estadios del } \\
\text { desarrollo }\end{array}$} & \multicolumn{8}{|c|}{ Huéspedes } \\
\hline & \multicolumn{2}{|c|}{$\begin{array}{l}\text { Odontesthes } \\
\text { mauleanum }\end{array}$} & \multicolumn{2}{|c|}{ Percichthys trucha } & \multicolumn{2}{|c|}{ Basilichthys australis } & \multicolumn{2}{|c|}{ Oncorhynchus mykiss } \\
\hline & $\mathrm{N}^{\mathrm{o}}$ & $\%$ & $\mathrm{~N}^{\mathrm{o}}$ & $\%$ & $\mathrm{~N}^{\mathrm{o}}$ & $\%$ & $\mathrm{~N}^{\mathrm{o}}$ & $\%$ \\
\hline $\mathrm{L}^{\mathrm{a}}$ & 20 & 4,7 & 1 & 20 & 1 & 2,9 & 6 & 28,6 \\
\hline $\mathrm{L} 4^{\mathrm{b}}$ & 147 & 34,3 & 1 & 20 & 9 & 25,7 & 4 & 19,0 \\
\hline $\mathrm{L} 3-\mathrm{L}^{\mathrm{c}}$ & 49 & 11,4 & 0 & 0 & 2 & 5,7 & 1 & 4,8 \\
\hline Machos & 52 & 12,1 & 0 & 0 & 6 & 17,1 & 3 & 14,3 \\
\hline Hembras inmaduras & 108 & 25,2 & 3 & 60 & 17 & 48,6 & 7 & 33,3 \\
\hline Hembras grávidas & 53 & 12,4 & 0 & 0 & 0 & 0 & 0 & 0 \\
\hline Total & \multicolumn{2}{|c|}{429} & \multicolumn{2}{|c|}{5} & \multicolumn{2}{|c|}{35} & \multicolumn{2}{|c|}{21} \\
\hline
\end{tabular}

Larvas del tercer estadio.

b Larvas del cuarto estadio.

c Larvas del tercer estadio mudando al cuarto estadio.

Cuadro 6. Estadios de desarrollo de Hysterothylacium geschei identificados en Odontesthes mauleanum en distintos meses en el lago Panguipulli, Chile.

Hysterothylacium geschei stages development identified in Odontesthes mauleanum in different months in Panguipulli Lake, Chile.

\begin{tabular}{|c|c|c|c|c|c|c|c|c|}
\hline \multirow{3}{*}{ Estadios } & \multicolumn{8}{|c|}{ Meses } \\
\hline & \multicolumn{2}{|c|}{ Septiembre 2006} & \multicolumn{2}{|c|}{ Enero 2007} & \multicolumn{2}{|c|}{ Marzo 2007} & \multicolumn{2}{|c|}{ Junio 2007} \\
\hline & $\mathrm{N}^{\circ}$ & $\%$ & $\mathrm{~N}^{\mathrm{o}}$ & $\%$ & $\mathrm{~N}^{\circ}$ & $\%$ & $\mathrm{~N}^{\circ}$ & $\%$ \\
\hline $\mathrm{L}^{\mathrm{a}}$ & 14 & 10,0 & 1 & 2,0 & 1 & 1,5 & 4 & 2,3 \\
\hline $\mathrm{L} 4^{\mathrm{b}}$ & 60 & 42,9 & 4 & 8,2 & 19 & 29,2 & 64 & 36,6 \\
\hline L3-L4 ${ }^{\mathrm{c}}$ & 7 & 5,0 & 4 & 8,2 & 4 & 6,2 & 34 & 19,4 \\
\hline Machos & 8 & 5,7 & 13 & 26,5 & 10 & 15,4 & 21 & 12 \\
\hline Hembras inmaduras & 47 & 33,5 & 11 & 22,4 & 18 & 27,7 & 32 & 18,3 \\
\hline Hembras grávidas & 4 & 2,9 & 16 & 32,7 & 13 & 20 & 20 & 11,4 \\
\hline Total & & & & & & & & \\
\hline
\end{tabular}

Larvas del tercer estadio.

b Larvas del cuarto estadio.

c Larvas del tercer estadio mudando al cuarto estadio.

Cuadro 7. Prevalencia, intensidad media y abundancia media de infección por Hysterothylacium geschei en Odontesthes mauleanum en distintos meses de muestreo en el lago Panguipulli, Chile.

Prevalence, mean intensity, and mean abundance of infection by Hysterothylacium geschei in Odontesthes mauleanum in different months in Panguipulli Lake, Chile.

\begin{tabular}{lccc}
\hline Meses & $\begin{array}{c}\text { Prevalencia } \\
\text { infectados/examinados } \\
(\%)\end{array}$ & Intensidad $^{\mathrm{a}}$ & $\begin{array}{c}\text { Abundancia } \\
\text { media }\end{array}$ \\
\hline Septiembre (S) & $7 / 9(78)$ & $20,4 \pm 32(2-83)$ & 15,6 \\
Enero (E) & $7 / 9(78)$ & $6,7 \pm 6,1(1-17)$ & 5,4 \\
Marzo (M) & $9 / 14(64)$ & $7,2 \pm 9,3(1-29)$ & 4,6 \\
Junio (J) & $5 / 12(42)$ & $34,8 \pm 36,4(8-94)$ & 14,6 \\
\hline
\end{tabular}

a Media \pm desviación estándar (mínima-máxima). Comparaciones de: 1) Prevalencia (probabilidad exacta de Fisher) $\mathrm{S} / \mathrm{E}(\mathrm{P}=1), \mathrm{S} / \mathrm{M}(\mathrm{P}=0,6), \mathrm{S} / \mathrm{J}$ $(\mathrm{P}=0,1), \mathrm{E} / \mathrm{M}(\mathrm{P}=0,7), \mathrm{E} / \mathrm{J}(\mathrm{P}=0,1), \mathrm{M} / \mathrm{J}(\mathrm{P}=0,9)$. Intensidad media, prueba de Kruskal-Wallis: $\mathrm{S} / \mathrm{E} / \mathrm{M} / \mathrm{J}: \mathrm{H}=3,5(\mathrm{P}>0,05), 3)$. Abundancia media S/E/M/J: H: 2,7 (P>0,05). 
que $O$. mykiss consume preferentemente Trichoptera (51\%), Aegla spp. (40\%), y peces (26\%) (Arenas 1978) en lagos y ríos de la cuenca del río Valdivia.

En O. mauleanum la prevalencia e intensidad y abundancia media de infección resultaron similares en los distintos muestreos correspondientes a diferentes estaciones. Por el contrario, la prevalencia y abundancia de infección por $H$. aduncum en $O$. mykiss y $O$. kisutch cultivados en Chiloé sugieren ser mayores en primavera (González 1998). En $H$. haze la prevalencia en A. fluvimanus alcanza un máximo de $70 \%$ en meses de otoño en la bahía de Tokio (Anderson 2000). Para H. bidentatum en Acipenser spp. del río Volga, la prevalencia es mayor en meses de verano y otoño (Moravec 1994). Las diferencias en los descriptores de la infección en distintos períodos podrían depender en parte de cambios en las características abióticas, densidad de hospederos intermediarios/paraténicos, dieta o respuesta del hospedero.

La prevalencia, intensidad media y abundancia media de infección por $H$. geschei en $O$. mauleanum, B. australis, $P$. trucha y $O$. mykiss no mostró asociación con el sexo del hospedero, coincidiendo con resultados observados en infecciones por Hysterothylacium incurvum e Hysterothylacium corrugatum en Xiphias gladius (García y col 2008). En algunos casos los descriptores de infección pueden resultar mayores en uno de los sexos del hospedero por diferencias en la frecuencia o abundancia de presas consumidas (Rohde 1993) u otros factores ligados al sexo como inmunocompetencia, tamaño corporal o comportamiento (Caillaud 2006). Al menos en O. mykiss en el lago Riñihue, unido al lago Panguipulli por el río Enco, ambos sexos presentan dieta similar (Arenas 1978).

La longitud estándar de los peces estuvo correlacionada significativamente con la abundancia de infección en $O$. mauleanum y $O$. mykiss; los peces de mayor talla presentaron mayor abundancia de infección. García y col (2008) no observaron correlación significativa entre intensidad de infección por $H$. incurvum e $H$. corrugatum y la talla de $X$. gladius. La existencia de correlación significativa podría atribuirse en parte a diferencias en la dieta existente entre peces de distinta longitud, consumiendo aquellos de mayor talla mayor diversidad o volumen de presas, incluidos hospederos intermediarios/paraténicos. Los cambios de dieta en diferentes tallas han sido observados en $O$. mauleanum (Klink y Eckmann, 1985), P. trucha y O. mykiss (Arenas 1978,1980). En el presente estudio $P$. trucha no mostró correlación entre longitud estándar e intensidad y abundancia de infección probablemente debido a que la talla de los peces analizados presentó un intervalo estrecho $(25-43 \mathrm{~cm}$, con un solo ejemplar de $16 \mathrm{~cm}$ ); de acuerdo con Arenas (1980), los peces de dicho intervalo consumen presas similares, esencialmente Aegla spp. y peces.

Hysterothylacium patagonense descrita en $P$. trucha en los lagos Aluminé y Huenchelican, en Argentina, parece establecerse con mayor facilidad que $H$. geschei en especies de salmónidos introducidos, ya que se han registrado prevalencias de $100 \%$ en $S$. trutta y $O$. mykiss y de $40 \%$ en $S$. fontinalis, pero siempre como estados larvarios (Moravec y col 1997). Al parecer, H. patagonense e $H$. geschei solo desarrollan hembras grávidas en $P$. trucha y $O$. mauleanum, respectivamente. Así, la trucha arco iris no tendría impacto en la diseminación y transmisión de la infección por $H$. geschei hacia los peces nativos. Por el contrario, H. aduncum desarrolla hembras grávidas en O. mykiss (González 1998), igual que el acantocéfalo Acanthocephalus tumescens, presente en Chile y Argentina, alcanzando esta última especie elevada proporción de parásitos grávidos en la trucha, contribuyendo a diseminar y favorecer la amplificación de la infección en los peces nativos en el lago Moreno, Argentina (Rauque y col 2003).

Aunque en el presente estudio no se identificaron larvas de $H$. geschei en los músculos de los peces examinados, se requieren trabajos experimentales para evaluar la capacidad de las L3 para invadir tal localización, así como también determinar su potencial riesgo de infección en mamíferos. Al menos en $H$. aduncum se comprobó que sus L3 pueden invadir los músculos de juveniles de Clupea harengus (Balbuena y col 2000) y de los estudios en músculos de peces consumidos en Chile, solo en $M$. magellanicus se observó Hysterothylacium sp. (Torres y col 2000, 2010, 2014). La localización muscular de $H$. aduncum podría explicar su asociación con cuadros alérgicos en los consumidores de pescado (Valero y col 2003) o el registro de un caso humano en Japón (Yagi y col 1996). También se ha comprobado experimentalmente que primates no humanos como el mono, Macaca mulatta, pueden desarrollar lesiones en el tracto digestivo al ingerir larvas de Hysterothylacium tipo MB descritas en peces marinos del golfo de México (Overstreet y Meyer 1981).

\section{AGRADECIMIENTOS}

Al Sr. Raúl Arriagada por su colaboración técnica en la captura de los peces y al Sr. Marcelo Delgado por su valiosa ayuda en el procesamiento de los peces en el laboratorio.

\section{REFERENCIAS}

Andersen K. 1993. Hysterothylacium aduncum (Rudolphi, 1862) infection in cod from the Oslofjord: seasonal occurrence of third and fourth stage larvae as well as adult worms. Parasitol Res 79, 67-72.

Anderson RC. 2000. Nematode parasites of vertebrates, their development and transmission. $2^{\text {nd }}$ ed. CAB International, Wallingford, UK.

Arenas JN. 1978. Análisis de la alimentación de Salmo gairdneri Richardson en el lago Riñihue y río San Pedro, Chile. Medio Amb 3, 50-55.

Arenas JN. 1980. Alimentación de Percichthys trucha (C \& V) en lagos y ríos valdivianos. Arch Biol Med Exp 11,163.

Balbuena JA, E Karlsbakk, A Kvenseth, M Saksvik, A Nylund. 2000. Growth and emigration of third-stage larvae of Hysterothylacium aduncum (Nematoda: Anisakidae) in larval herring Clupea harengus. J Parasitol 86, 1271-1275.

Brizzola SM, RD Tanzola. 1995. Hysterothylacium rhamdiae sp. n. (Ascaridoidea: Anisakidae) from a Neotropical catfish, Rhamdia sapo (Pisces: Pimelodidae). Mem Inst Oswaldo Cruz 90, 349-352. 
Caillaud D, F Prugnolle, P Durand, A Théron, T Meeus. 2006. Host sex and parasite genetic diversity. Microb Infect 8, 2477-2483.

Campos H. 1985. Distribution of the fishes in the Andean rivers in the South of Chile. Arch Hydrobiol 104, 169-191.

Campos H, W Steffen, G Agüero, O Parra, L Zúñiga. 1992. Limnology of Lake Ranco, Chile. Limnologica 22, 337-353.

Campos H. 2000. Estudio de los Lagos Neltume y Pirehueico. Informe Final. Instituto de Zoología/Dirección General de Aguas, Departamento de Estudios y Planificación. Ministerio de Obras Públicas, Chile. Pp 1-258.

Fagerholm HP. 1982. Parasites of fish in Finland. Acta Acad Aboensis Ser B 40, 1-128.

Golusda P. 1927. Aclimatación y cultivo de especies salmonídeas en Chile. Bol Soc Biol Concepción 1, 80-100.

García A, MN Santos, S Damiano, G Nascetti, S Mattiucci. 2008. The metazoan parasites of swordfish from Atlantic tropical - equatorial waters. J Fish Biol 73, 2274-2287.

González L, J Carvajal. 1995. New host records of larval Hysterothylacium aduncum (Nematoda: Anisakidae) in fauna associated with salmonid sea farms in Chile. Res Rev Parasitol 55, 247-253

González L. 1998. The life cycle of Hyterothylacium aduncum (Nematoda: Anisakidae) in Chilean marine farms. Aquaculture 162, 173-186.

Hurst RJ. 1984. Marine invertebrate hosts of New Zealand Anisakidae (Nematoda). New Zealand J Mar Fresh Res 18, 187-196.

Kelly DW, RA Patterson, CR Townsend, R Poulin, DM Tompkins. 2009. Has the introduction of brown trout altered disease patterns in native New Zealand fish? Freshwater Biol 54, 1805-1818.

Kennedy CR, AO Bush. 1994. The relationship between pattern and scale in parasite communities: a stranger in a strange land. Parasitology 109, 187-196

Klimpel S, S Ruckert. 2005. Life cycle strategy of Hysterothylacium aduncum to become the most abundant anisakid fish nematode in the North Sea. Parasitol Res 97, 141-149.

Klink A, R Eckmann. 1985. Age and growth, feeding habits, and reproduction of Cauque mauleanum (Steindachner 1896) (Pisces: Atherinidae) in Southern Chile. Stud Neotrop Fauna Environm 20, 239-249.

Knoff M, NN Felizardo, AM Íñiguez, EJL Torres, RM Pinto, DC Gómez. 2012. Genetic and morphological characterization of a new species of the genus Hysterothylacium (Nematoda) from Paralichthys isosceles Jordan, 1890 (Pisces: Teleostei) of the Neotropical Region, State of Rio de Janeiro, Brazil. Mem Inst Oswaldo Cruz 107, 186-193.

Koie M. 1993. Aspects of the life cycle and morphology of Hysterothylacium aduncum (Rudolphi, 1802) (Nematoda, Ascaridoidea, Anisakidae). Can J Zool 71, 1289-1296.

Li L, LP Zhang, YY Liu. 2013. Hysterothylacium simile n. sp. and $H$. aduncum (Rudolphi, 1802) (Nematoda: Raphidascarididae) from marine fishes in the Bohai and Yellow Sea, China, with comments on the record of $H$. paralichthydis (Yamaguti, 1941) from Chinese waters. Syst Parasitol 84, 57-69.

Marcogliese DJ. 1996. Larval parasitic nematodes infecting marine crustaceans in eastern Canada. 3. Hysterothylacium aduncum. $J$ Helminthol Soc Wash 63, 12-18.

Margolis L, GW Esch, JC Holmes, AM Kuris, GA Schad.1982 The use of ecological terms in Parasitology (report of an ad hoc committee of the American Society of Parasitologists). J Parasitol 68, 131- 133.

Molnar K, K Buchmann, C Székely. 2006. Phylum Nematoda. In: Woo PTK (ed.). Fish Diseases and Disorders. Volume 1 Protozoan and Metazoan Infections. CABI, Wallingford, UK, Pp 417-443.

Moravec F. 1994. Parasitic Nematodes of Freshwater Fishes of Europe. Kluwer Academic Publishers, Dordrecht, Czech Republic.

Moravec F, S Urawa, CO Coria. 1997. Hysterothylacium Patagonense n. sp. (Nematoda: Anisakidae) from freshwater fishes in Patagonia, Argentina, with a key to the species of Hysterothylacium in American freshwater fishes. Syst Parasitol 36, 31-38.

Norris DE, RM Overstreet. 1976. The public health implications of larval Thynnascaris nematodes from shellfish. J Milk Food Technol 39, 47-54.
Overstreet RM, GW Meyer. 1981. Hemorrhagic lesions in stomach of rhesus monkey caused by piscine ascaridoid nematode. J Parasitol 67, 226-235.

Poulin R, D Mouillot. 2003. Host introductions and the geography of parasite taxonomic diversity. J Biogeogr 30, 837-845.

Rauque CA, GP Viozzi, LG Semenas. 2003. Component population study of Acanthocephalus tumescens (Acanthocephala) in fishes from Lake Moreno, Argentina. Folia Parasitol 50, 72-78.

Rohde K. 1993. Ecology of Marine Parasites. CAB International,Wallingford, UK.

Ruiz VH, M Marchant. 2004. Ictiofauna de Aguas Continentales Chilenas. Universidad de Concepción, Concepción, Chile.

Rye LA, MR Baker. 1992. The life history of Hysterothylacium analarum Rye and Baker, 1984 (Nematoda: Anisakidae) in Lepomis gibbosus (Pisces: Centrarchidae) in Southern Ontario, Canada. Can J Zool 70, 1576-1584.

Sachs L. 1968. Estadística aplicada. Editorial Labor, Madrid, España.

Salinas X, MT González, E Acuña. 2008. Metazoan parasites of the thumb grenadier Nezumia pulchella, from the South-Eastern Pacific, off Chile, and their use for discrimination of host populations. $J$ Fish Biol 73, 683-691.

Sepúlveda F, SL Marín, J Carvajal. 2004. Metazoan parasites in wild fish and farmed salmon from aquaculture sites in Southern Chile. Aquaculture 235, 89-100.

Siegel, S. 1972. Estadística no Paramétrica. Editorial Trillas, México D. F.

Soto D, H Campos. 1996. Los lagos oligotróficos del bosque templado húmedo del sur de Chile. En: Armesto JJ, Villagrán C, Arroyo MK (eds.). Ecología de los bosques nativos de Chile. Editorial Universitaria, Santiago, Chile, Pp 317-334.

Torres P, C Jara. 1986. Aegla denticulata (Crustacea: Decapoda): un nuevo huésped para Hysterothylacium sp. (Nematoda: Anisakidae) en el sur de Chile. Parasitol al Día 10, 134-135.

Torres P, J Arenas, A Neira, X Cabezas, C Covarrubias, C Jara, C Gallardo, M Campos. 1988. Nematodos anisákidos en peces autóctonos de la cuenca del río Valdivia, Chile. Bol Chil Parasitol 43, 37-41.

Torres P. 1995. Some trematode, nematode, and acanthocephalan parasites of rainbow trout, Oncorhynchus mykiss introduced into Chile. $J$ Helminthol Soc Wash 62, 257-259.

Torres P, P Andrade, R Silva.1998. On a new species of Hysterothylacium (Nematoda: Anisakidae) from Cauque mauleanum (Pisces: Atherinidae) by brightfield and scanning electron microscopy. Mem Inst Oswaldo Cruz 93, 745-752.

Torres P, R Moya, J Lamilla. 2000. Nematodos anisákidos de interés en salud pública en peces comercializados en Valdivia, Chile. Arch Med Vet 32, 107-113.

Torres P, MS Soto. 2004. Hysterothylacium winteri sp. n. (Nematoda: Anisakidae), a parasite of Chilean rock cod, Eleginops maclovinus (Perciformes: Eleginopidae), from South Chile. Folia Parasitol $51,55-60$

Torres P, C Quintanilla, M Rozas, P Miranda, R Ibarra, MF San Martín, B Raddatz, M Wolter, A Villegas, C Canobra, M Hausdorf, R Silva. 2010. Endohelminth parasites from salmonids in intensive culture from southern Chile. J Parasitol 96, 669-670.

Torres P, S Puga. 2011. Comparative efficacy of candling and glass plate compression for detection of diphyllobothriosis in rainbow trout (Oncorhynchus mykiss) musculature. Rev Sci Tech Off Int Epiz 30, 831-837.

Torres P. 2012. Parásitos eucarióticos de peces y su importancia en la salud. In: Canals M, PE Cattan (eds). Zoología Médica III. Vertebrados. Editorial Universitaria, Santiago, Chile, Pp 213-265.

Torres P, L Castillo, S Puga, J Lamilla, JC Miranda. 2014. Helmintos, myxozoos y microsporidios en músculos de peces comercializados frescos y su importancia como riesgo potencial para la salud humana en la ciudad de Valdivia, Chile. Arch Med Vet 46, 83-92.

Valero A, S Terrados, V Díaz, V Reguera, J Lozano. 2003. Determination of IgE in the serum of patients with allergic reactions to four species of fish parasite anisakids. J Invest Allergol Clin Immunol 13, 94-98. 
Yagi K, K Nagasawa, H Ishikura, A Nagasawa, N Sato, K Kikuchi, H Ishikura. 1996. Female worm Hysterothylacium aduncum excreted from human: a case report. $J p$ J Parasitol 45, 12-23.

Yoshinaga T, K Ogawa, H Wakabayashi. 1987. Experimental life cycle of Hysterothylacium aduncum (Nematode: Anisakidae) in fresh water. Fish Pathol 22, 234-251.
Yoshinaga T, K Ogawa, H Wakabayashi. 1989. Life cycle of Hysterothylacium haze (Nematoda: Anisakidae: Raphidascaridinae). J Parasitol 75, 756-763.

Zar JH. 1999. Biostatistical Analysis. $4^{\text {th }}$ ed. Prentice-Hall, Upper Saddle River, New Jersey, USA. 